\title{
Correction: In-vitro antidiabetic activities, chemical compositions, antioxidant activities, and toxicity of black tea polysaccharides as a potential source of dietary ingredients
}

\author{
Ebru Pelvana, Ayse Karadag ${ }^{\mathrm{b}}$, Kubra Dogan ${ }^{\mathrm{b}}, \mathrm{Soner} \mathrm{Aksu}^{\mathrm{c}}$, Arzu Tas ${ }^{\mathrm{c}}$, Kubra Akalın ${ }^{\mathrm{d}}$, Özlem Atlı \\ Eklioğlu ${ }^{\mathrm{e}}$ and Cesarettin Alasalvar ${ }^{\mathrm{a}^{*}}$
}

${ }^{a}$ TÜBITTAK Marmara Research Center, Food Institute, P.O Box 21, 41470 Gebze-Kocaeli, Turkey

bDepartment of Food Engineering, Yıldız Technical University, 34210 Esenler-Istanbul, Turkey

'TÜBİTAK Marmara Research Center, Genetic Engineering and Biotechnology Institute, P.O Box 21, 41470 Gebze-Kocaeli, Turkey

dDepartment of Molecular Biology and Genetics, Institute of Science and Technology, Gebze Technical University, 41400 Gebze-Kocaeli, Turkey

eDepartment of Pharmaceutical Toxicology, Faculty of Pharmacy, Anadolu University, 26470 Tepebaş1-Eskişehir, Turkey

*Corresponding author: Cesarettin Alasalvar, TÜBITAK Marmara Research Center, Food Institute, P.O Box 21, 41470 Gebze-Kocaeli,

Turkey. E-mail: cesarettin.alasalvar@tubitak.gov.tr

DOI: $10.31665 / \mathrm{JFB} .2021 .14275$

Received: April 15, 2021; Revised received \& accepted: April 16, 2021

Citation: Pelvan, E., Karadag, A., Dogan, K., Aksu, S., Tas, A., Akalın, K., Eklioğlu, Ö.A., and Alasalvar, C. (2021). Correction: In-vitro antidiabetic activities, chemical compositions, antioxidant activities, and toxicity of black tea polysaccharides as a potential source of dietary ingredients. J. Food Bioact. 14: 126-127.

There is an error in Table 2 in the published article, Journal of Food Bioactives, 2021;13:93-101, doi: 10.31665/JFB.2021.13263. The correct value in column " $\mathrm{mg} / 100 \mathrm{~g}$ " under "D-glucose" is " $59.50 \pm 7.80$ ", not " $9.50 \pm 7.80$ ". The authors have included a corrected version of the table below. 
Antidiabetic activity and toxicity of black tea polysaccharides

Table 2. Monosaccharide, amino acid, and mineral compositions of BTPS

\begin{tabular}{lllclc}
\hline Monosaccharides & mol ratio (\%) & Amino acids & mg/100 g & Minerals & mg/100 g \\
\hline D-mannose & $2.38 \pm 0.02$ & Aspartic acid & $446 \pm 86$ & Calcium & $41.2 \pm 1.7$ \\
D-ribose & $2.72 \pm 0.02$ & Alanine & $114 \pm 11$ & Chromium & $0.78 \pm 0.0$ \\
L-rhamnose & $2.86 \pm 0.03$ & Arginine & $89.5 \pm 12$ & Copper & $1.37 \pm 0.0$ \\
D-glucuronic & $2.46 \pm 1.01$ & Glutamic acid & $822 \pm 11$ & Iron & $9.8 \pm 0.2$ \\
D-galacturonic & $2.89 \pm 1.02$ & Glycine & $337 \pm 60$ & Magnesium & $815 \pm 27$ \\
D-glucose & $7.00 \pm 0.07$ & Histidine & $59.50 \pm 7.80$ & Manganese & $423 \pm 4$ \\
D-galactose & $47.5 \pm 0.5$ & Isoleucine & nd & Phosphorus & $1,970 \pm 50$ \\
D-xylose & $1.89 \pm 0.02$ & Leucine & $54.5 \pm 3.5$ & Potassium & $9,155 \pm 47$ \\
D-arabinose & $28.5 \pm 0.3$ & Lysine & $182 \pm 12$ & Sodium & $15.3 \pm 0.8$ \\
L-fucose & $1.45 \pm 0.08$ & Methionine & $\mathrm{nd}$ & Zinc & $6.22 \pm 0.20$ \\
& & Proline & $85.0 \pm 9.9$ & & \\
& & Phenylalanine & $20.0 \pm 4.2$ & & \\
& & Serine & $144 \pm 12$ & & \\
& & Threonine & $67.0 \pm 0.0$ & & \\
& & Tyrosine & $40.0 \pm 14.1$ & & \\
\end{tabular}

Data are expressed as the mean $\pm \mathrm{SD}(n=3)$. Abbreviation: $n d$, not detected. 